\title{
Commercial Availability, Safety, and Hazards
}

DANA L. ROTH

California Institute of Technology, Millikan 1-32, Pasadena, CA 91125, US Email: dzrlib@library.caltech.edu

\subsection{INTRODUCTION}

The importance of being able to obtain chemicals commercially, being aware of necessary laboratory precautions, and having access to hazardous-property information is essential for students and researchers. This chapter provides commercial availability links to individual chemical company catalogs and to websites that are linked to multiple commercial sources. It describes laboratory-safety information resources and provides hazardous-property information, for both individual and classes of chemicals, and includes appropriate search techniques.

Information on commercial availability, safety, and hazards is interrelated in the sense that commercial chemical companies are responsible for providing Material Safety Data Sheets (MSDSs) for their products. The MSDS for a substance provides basic physical-property information, possible hazardous properties, and instructions for safe use.

\subsection{COMMERCIAL AVAILABILITY}

Individual supplier catalogs, such as Aldrich Chemistry, as well as many catalogs including the products of multiple suppliers, such as Chemical 
Book, are freely available online. In addition, both SciFinder and Reaxys provide subscribers with links from individual compound records to several suppliers or supplier catalogs. Given the enormous number of supplier websites for common chemicals (e.g., search Google for "suppliers of benzene"), serious consideration should be given to finalizing searches using well-established, up-to-date, individual supplier catalogs, since some suppliers do not actually stock chemicals but claim to make them on demand.

In general, use of CAS Registry Numbers is recommended, since they are unique, rather than chemical names or molecular formulas, which can be ambiguous. It should be noted that each stereochemical or isotopic variant of a compound has a unique CAS Registry Number (CAS RN).

\subsubsection{Commercial Availability Resources}

7.2.1.1 Individual Company Sites. Aldrich Chemistry ${ }^{1}$ offers a full range of chemistry products for a variety of research areas, including asymmetric syntheses, catalysis, chemical biology, greener alternatives, organometallics, solvents, stable isotopes, etc. This website also includes products from the Fluka and Sigma lines. The catalog is both text and structure searchable. The default text search is limited to either product names/numbers or CAS Registry Numbers. Structure searching offers the option of three structure editors (JME, Marvin, ChemDraw), plus the ability to limit by ranges of MW, BP, or MP. The advanced search offers a wide variety of "search type" and "search method" options for text, plus the ability to perform keyword searches of the Sigma-Aldrich web pages, PDFs, DataSheets, technical bulletins, brochures, etc.

The product records provide a link for downloading the Material Safety Data Sheet for the substance (which presumably updates the 1988 Sigma-Aldrich library of chemical safety data), and generally include brief physical property data, safety information, references to the Merck Index, Beilstein, Fieser \& Fieser, and the Aldrich spectra catalogs. They also provide a links out to PubChem Substance and may link to PDF images of IR or NMR spectra. In addition to a listing of research grades, there may also be a link to bulk ordering and pricing options.

7.2.1.2 Multiple Supplier Sites. Chemical $\mathbf{B o o k}^{2}$ is a freely available site for commercial chemicals, with options for an English, Chinese, German, or Japanese interface. The default search is "Products", which is searchable with a CAS Registry Number or a chemical name/ synonym. Since companies are not always careful about assigning the current CAS RN, it may be necessary to perform a second search 
using a chemical name (for example, Pyrogallol Red) to ensure complete results.

The "Chemical site" option provides short descriptions of the nearly 50 companies, most of which are located in China. Chemical products are also browsable by category (e.g., Amino Acid Derivatives, Catalysis \& Inorganic Chemistry, etc.). It is also possible to browse CAS Registry Numbers, the chemical name index or the company website index (which lists thousands of international companies and gives links to a seemingly random listing of their chemical products).

Search results include a structural diagram, basic physical properties, and links to both China and global suppliers, chemical properties ( basic information, physical properties, safety information, usage and synthesis, preparation products and raw materials, etc.) and MSDS providers.

$\mathrm{Chem} \mathrm{Net}^{3}$ is a freely searchable listing of chemicals and related products from over 26,000 worldwide suppliers. The default home page "Product Search" box allows searching by product name, CAS RN, or molecular formula. Data for individual compounds, however, do not seem as extensive as Chemical Book. Additional search options are Company Search (keyword searchable), ChemSearch (keyword web search), and Directory Search (keyword site search).

In addition to the ChemNet home page, there are tabs for pages that allow one to browse or search through products, suppliers, offers to buy and sell, chemical news, REACH, and CAS RNs. The "Resource" tab has a list of links to chemical categories, such as colour index, drug synthesis, FEMA (Flavor chemicals) index, Open Access journals, pesticide common names, and FDA databases.

MatWeb ${ }^{4 a}$ provides searchable data sheets for over 90,000 polymers, plastics, metals, alloys, ceramics, semiconductors, fibers, and engineering materials. Each data sheet provides an extensive listing that includes a material description, physical, mechanical, thermal, and processing properties. MatWeb allows searchers to identify suppliers for both specific materials and discovered materials with specific properties.

Free registration is recommended in general, ${ }^{4 \mathrm{~b}}$ but it is required to access the Advanced Search Engine, which offers iterative searching. Search options include text, property data (physical, mechanical, etc.), material category (carbon, ceramic, metal, etc.). The advanced search option is extremely powerful; for example, one can use it to perform a search for substances that are alloys, have tensile stresses within a specific range of values, and contain a nonferrous metal.

MatWeb also includes several other search tabs. "Category" allows you to browse and search by material categories. "Property" offers a combination search of material category and material properties, which 
includes links to "Polymer Film Search" and "Lubricant Search". "Metals" presents a combination search of material category and material composition. The remaining tabs, "Trade Name" and "Manufacturer," are self-explanatory.

The ChemExper Chemical Directory ${ }^{5}$ is a freely available catalog consisting of more than 9 million chemicals from more than 2,300 suppliers. The default "Quick Search" accepts molecular formula, CAS Registry Numbers, product names or synonyms, and SMILES strings. Structure searching, both exact and substructure, is available with the JME Molecular Editor. The "Advanced Search" option offers a combination of IUPAC name (equals, starts with, contains), molecular formula, and structure, as well as inequality and range relations for numeric fields, such as boiling point, melting point, density, and IR absorption frequencies. Availability of NMR spectra can also be specified. Records for specific chemicals include the following information: CAS RN, molecular formula, molecular weight, boiling point, density, cLogP, cLogS, polar surface, 3D model viewer, InChIKey, etc.

SciFinder $^{6}$ (subscription required) has links, in REGISTRY, to CHEMCATS, a database that indicates commercial availability of substances. At the time of this writing, CHEMCATS has more than 67 million products (based on $\sim 20$ million unique chemical substances) found in $\sim 1,000$ supplier catalogs. While some records do include quantities and pricing, others do not, and it is always wise to contact the supplier directly for the most up-to-date pricing, availability, quantities, purities, etc.

Reaxys $^{7}$ (subscription required) has links, indicated by a flask icon under the structural diagram, to Accelry's ACD (license required), eMolecules (freely available), and Cambridge Soft ChemACX (subscription required). eMolecules ${ }^{8}$ can also be freely searched directly using either chemical structures or names. Pricing and use of the database for screening purposes requires a subscription.

\subsection{SAFETY}

\subsubsection{The Importance of Safety in the Chemical Laboratory}

The American Chemical Society issued "The Chemical Professional's Code of Conduct" in 2007. It lists a number of safety-related issues, specifically, that chemical professionals should "actively be concerned with the health and safety of co-workers, consumers and the community," that "they should strive to understand and anticipate 
the environmental consequences of their work," and that "they have a responsibility to minimize pollution and to protect the environment"?

Unfortunately, readers of Chemical \& Engineering News are reminded, on an almost weekly basis, of violations of the "Code of Conduct" in both the "Chemical Safety" Letters to the Editor ${ }^{10}$, and in Jyllian Kemsley's "The Safety Zone" blog, ${ }^{11}$ which provides a "Friday Chemical Safety Round-up," including sections on chemical health and safety news from the past two weeks, fires and explosions, leaks, spills, and other exposures. Most of the time, however, it does not specifically include stories related to meth labs; ammonia leaks; incidents involving floor sealants, cleaning solutions, or pool chemicals; transportation spills; and fires from oil, natural gas, or other fuels. In addition to the Safety Round-up, the "Safety Zone" has several other sections, including Popular Posts, Recent Posts, Recent Comments, Lessons Learned Reports, Blogroll, and Chemical Safety Resources.

This dichotomy, between the "Code" and the "Safety Zone" reports, strongly suggests that everyone working with chemicals should increase their awareness of possible hazards in their work, employing both print and electronic resources to learn more about both the dangers of working with chemicals and protective measures that should be taken. The pervasive nature of the general problem of safety, especially in academia, was recently recognized by the ACS Committee on Chemical Safety (CCS), which recently convened a task force that produced a draft report, "Creating Safety Cultures in Academic Institutions,", 12 which is available on the CCS website. This draft report is focused on identification of elements of strong safety cultures, safety education, and enhancement of safety cultures.

\subsubsection{Safety Resources}

In addition to developing a general culture of safe handling of chemicals, it is also essential to maintain an on-going awareness of statutory regulations related to their use. Two essential publications help meet these needs; Material Safety Data Sheets, ${ }^{13}$ available from chemical suppliers, ${ }^{14}$ provide safe-handling procedures, health hazards, exposure limits, and basic physical properties, while the very practical publication "Prudent Practices in the Laboratory: Handling and Disposal of Chemicals" 15 has chapters on "The Culture of Laboratory Safety", "Working with Chemicals", "Government Regulation of Laboratories", and other, general topics related to laboratory safety. 
In addition to freely available electronic resources, there are also a variety of print resources, easily available in many university and large public libraries, including:

R. H. Hill, Jr., D. C. Finster, Laboratory safety for chemistry students, Wiley, 2010.

A. K. Furr, CRC handbook of laboratory safety, $5^{\text {th }}$ edition, CRC Press, 2000.

On being a scientist: A guide to responsible conduct in research. National Academies Press, 2009.

http:/ www.nap.edu/catalog.php?record_id=12192, Accessed May 24, 2013.

R. Montesano ... W. Davis, Handling chemical carcinogens in the laboratory: Problems of safety, IARC Scientific Publications; no. 33, Who Publications Center, 1979.

Safety in academic chemistry laboratories, $7^{\text {th }} \mathrm{ed}$., American Chemical Society, Joint Board-Council Committee on Chemical Safety, 2003. Volume 1: Accident prevention for college and university students, http:/portal.acs.org/portal/PublicWebSite/about/ governance/committees/chemicalsafety/publications/ WPCP_012294, Accessed May 24, 2013.

Volume 2: Accident prevention for faculty and administrators, http://www.chem.uoa.gr/misc/SACL_faculty.pdf, Accessed May 24, 2013.

N. V. Steere, Safety in the chemical laboratory, v.1-4. Reprinted from J. Chem. Educ., American Chemical Society Division of Chemical Education, 1967-1981.

It can be challenging to effectively search for the most up-to-date information on hazards and safety; the Chemical Information Sources ${ }^{16}$ wiki book provides additional tips on performing such searches for safety information, and any librarian or information professional will also be able to provide assistance in this area.

\subsection{HAZARDS}

\subsubsection{Introduction}

The Chemical Abstracts Service currently adds approximately 15,000 new substances to its Registry File each day, ${ }^{17}$ and controversies over the assessment of chemical risks in the United States continue. While only relatively few of these substances will enter the environment, 
current federal regulations are widely recognized as inadequate, especially for nanoparticles and hormone mimics, ${ }^{18}$ two classes of compounds that are especially insidious since, while they may not be toxic, they can have deleterious effects on pulmonary function and fetal development.

The Toxic Substances Control Act, ${ }^{19}$ which became law in 1976, regulates the introduction of new or existing chemical substances in the market place. It is flawed, however, in the sense that it did not provide a mechanism for dealing with the possible toxicity of substances already on the commercial market. The Act mandates that the EPA maintain the TSCA Chemical Substance Inventory, ${ }^{20}$ which is a listing of existing chemicals. Chemicals not listed generally must be reviewed by the EPA prior to their commercial use.

This review process, however, is questionable and apparently subject to external pressures. A Chicago Tribune ${ }^{21}$ investigation of flame retardant chemicals suggests that these pose significant health risks and are generally ineffective. It also identifies highly questionable legislative testimony regarding the necessity of adding toxic chemicals to products, especially those destined for infants.

Recent positive developments include the EPA's expansion of the use of computational toxicology in regulatory decisions about commercial chemicals and pesticides, as well as unregulated contaminants in drinking water. This effort, called "ToxCast", focuses on how chemicals cause health effects and promises to reduce the use of animal testing. ${ }^{22}$

\subsubsection{Nano Particles. Nano particles are of special concern} because their hazard potential was originally based on the material's bulk properties. This unfortunate situation is only recently being recognized, and steps are rapidly being taken to fully explore the difference in the chemical nature of, for example, gold nanoparticles as contrasted with metallic gold. ${ }^{23}$

The remarkable diversity of new nanomaterials is causing serious consideration of reinventing toxicological testing. ${ }^{24}$ Currently, there is an enormous variety of potential nanomaterials, but no standards for determining which are possibly toxic to humans and other species. This situation is an obvious threat to public confidence in new products and, if continued, will certainly restrict their development. Standard toxicological approaches, which require testing of individual materials, are thought to be an inadequate response to the demands in the current economic climate; so, new approaches are being tested. ${ }^{25}$ In 2012, the current state of the art of both the science and the significant data gaps posed by engineered nanomaterials was described in a National 
Research Council publication entitled, "A research strategy for environmental, health, and safety aspects of engineered nanomaterials."26

An additional problem that arises when attempting to retrieve toxicity information about nanoparticles is that they have only recently been identified as such in the Chemical Abstracts Service's CAplus records. The CAS Registry does not separately register either nanomaterials or nanoscale objects (as defined by the external dimensions of the material). These materials are indexed with the same CAS RN as the bulk material. This leads to a complicated search strategy; for example, searching Chemical Abstracts for the toxicity of a beryllium nanoparticle on SciFinder requires a search for the element (e.g., Substance Identifier $=$ Beryllium; CAS $\mathrm{RN}=7440-41-7$, etc.), retrieving the references limited by the substance role (Adverse Effect, including toxicity), and then refining by research topic with the word "nano".

In SciFinder/STN, a NANO super role has been added for those substances, which have been indexed in records since 1992, if they are described as being either:

- in a nano form or as nanoscale without further information; or

- between 0.05 and 100 nanometers in one dimension.

Tox Town, the National Library of Medicine's interactive guide to commonly encountered toxic substances, is a resource that provides general information, and has released a Nanoparticles page. ${ }^{27}$

7.4.1.2 Hormone Mimics. Hormone mimics are another serious problem. A fairly detailed account of the effects of estrogenic mimics is given in the Wikipedia article on Endocrine disruptors. ${ }^{28} \mathrm{~A}$ recent study, ${ }^{29}$ funded by the National Institute of Environmental Health Sciences, quantified the biological effects of estrogenic activity, and found that about 95 percent of commercial plastic items tested positive for estrogenic activity, although not all are toxic. As pointed out in a recent review,

"Exposure in people is typically a result of contamination of the food chain, inhalation of contaminated house dust or occupational exposure. EDCs include pesticides and herbicides (such as dichlorodiphenyl trichloroethane or its metabolites), methoxychlor, biocides, heat stabilizers and chemical catalysts (such as tributyltin), plastic contaminants (e.g., bisphenol A), pharmaceuticals (i.e., diethylstilbestrol; $17 \alpha$-ethinylestradiol) or dietary components (such as phytoestrogens)". 30 
Sources of information for hazardous materials are widely varied. Academics provide the basic research which is reported in journal articles and comprehensively indexed in PubMed, ${ }^{31}$ industrial organizations that have concerns about the health of their employees and any legal obligations governing both industrial use and commercial products require access to a variety of database resources, and a wide variety of government agencies have statutory responsibilities for both research and enforcement of regulations, as reflected in the Federal Register, ${ }^{32}$ which is excerpted in ChemList (subscription required). ${ }^{33}$

\subsubsection{Hazards Resources}

Searching for information on hazardous chemicals is generally very straightforward, due to the universal use of Chemical Abstracts Registry Numbers for compound identification. Fortunately, there are a wide variety of both subscription and freely available databases, which update and continue many print resources that remain useful for the compounds they describe.

\subsubsection{Hazards Resources - Subscription Required. Bretherick's} Handbook of Reactive Chemical Hazards ${ }^{34}$ covers some 5,000 elements and compounds. Information on the instability of individual compounds and hazardous interactions between elements and/or compounds appears in volume one; classes, groups of elements, and compounds possessing similar structures or hazard potential are alphabetically listed in volume two. Entries are in chemical formula order, with extensive cross-indexing. Source material is based on both journal articles and book series on synthetic techniques. These sources are supplemented by encyclopedia works such as Mellor, Houben-Weyl, and Kirk-Othmer, as well as Chemical Abstracts, and the two RSC publications, Laboratory Hazards Bulletin and Chemical Hazards in Industry. Print, Kindle, and other online versions of the handbook are available, and Bretherick's forms the foundation of Elsevier's HazMat Navigator database. The following appendices are included.

- Source title abbreviations used in references.

- Tabulated fire-related data.

- Glossary of abbreviations and technical terms.

- Index of chemical names and synonyms used in section 1.

- Index of class, group and topic titles used in section 2.

- Index of section 2 titles classified by type.

- Index of CAS registry numbers vs. serial numbers in section 1 . 
Hazmat Navigator ${ }^{35}$ is a chemical safety database based on Bretherick's Handbook of Reactive Chemical Hazards, with additional content from Sittig's Handbook of Toxic and Hazardous Chemicals and Carcinogens, Sittig's Handbook of Pesticides and Agricultural Chemicals, Encyclopedia of Toxicology, and the Fire Protection Guide to Hazardous Materials. In addition to critical, detailed chemical hazards information from Bretherick's, Hazmat Navigator includes information on physical properties, handling, safety, flammability and toxicity from both the journal literature and other resources. This information is updated annually.

The search page offers the following search options:

- Quick: chemical name or synonym, CAS RN or molecular formula.

- Advanced: keyword database search or fielded search.

- Structure/substructure: draw structures using the MarvinSketch structure editor.

- Index: alphabetical listing of contents with links.

The element/compound record provides links to:

- Properties: names, properties, RTECS \#, Code \#s, emolecules, etc.

- Handling: recommendations for PPE, spill, storage, disposal, etc.

- Safety: common uses, first aid, exposure guidelines, etc.

- Reactivity: common reactive hazards, links to related records.

- Flammability: fire and explosion hazards, firefighting procedure.

- Toxicity: human acute toxicity, target organs, environmental fate, etc.

A demonstration video with scrollable sample pages from the Benzene record is available at http:/tinyurl.com/ych68rk.

An interesting and very important feature of Hazmat Navigator is the link to related records. For example, the record for Benzvalene (a very strained Benzene isomer), has only partial information (i.e., a brief listing of properties and a short reactive hazard note), but it also has a related record link to Strained-Ring Compounds, a listing of similar compounds and compound groups with Hazmat records.

Sax's Dangerous Properties of Industrial Materials ${ }^{36}$ currently lists more than 26,000 substances, an increase of approximately $10 \%$ over the previous edition. In addition, approximately 1,000 chemicals have been updated to include IDLH (Immediately Dangerous Life or Health) levels. Sax is unique in the number of chemicals listed and the breadth of information on toxic and mutagenic potential, flammable and explosive 
properties, reactivity and physical properties, and regulatory data. Print indexes include synonym lists and CAS Registry Numbers. Sax is available in print, on CD-ROM, on the Wiley Online Library, and in Knovel. Wiley Online search options include full text, article title, CAS $\mathrm{RN}$, and chemical name

Patty's Industrial Hygiene and Toxicology Online ${ }^{37}$ is the electronic version of the sixth edition of Patty's Industrial Hygiene and the Sixth edition of Patty's Toxicology. Patty's Industrial Hygiene is focused on the engineering control of exposure to chemical, physical, and biological hazards in the workplace. Patty's Toxicology provides toxicological information for chemical compounds used in industry, including metals, organics, and polymers. Patty's online is available in the Wiley Online Library, searchable as combined or separate volumes, and in Knovel. Wiley Online search options include full text, article title, CAS RN or chemical name, and topic.

Dictionary of Substances and their Effects (DOSE) ${ }^{38}$ covers over 4,100 chemicals, including pesticides, food carcinogens, and endocrine disruptors. Information is provided on physical properties, exposure limits, and toxicity in general, as well as geno- and ecotoxicity, environmental fate, and regulatory requirements. The second edition appeared in seven volumes in 1999, and purchase of the print volumes includes site-wide access to DOSE Online, a fully searchable web database. Knovel, however, provides access to the third edition (2005), which covers $\sim 5,300$ compounds. Search options include chemical name/synonym, CAS RN, and EINECS/RTECS number. The RSC website, http:// www.rsc.org/Publishing/CurrentAwareness/DOSE/SampleRecords.asp, provides a sample record.

\subsection{Chemical Hazard Information Library. The Chemical} Hazard Information Library $(\mathrm{CHIL})^{39}$ is an online collection of full text documents from over 100 national and international sources, including: EPA (Environmental Protection Agency), NLM (National Library of Medicine), EU (European Union), WHO (World Health Organization, NTP (National Toxicology Program), ATSDR (Agency for Toxic Substances and Disease Registry), NCI (National Cancer Institute), IARC (International Agency for Research on Cancer), DoD (Department of Defense), etc. It contains toxicology and related hazard data affecting both humans and the environment. The complexity of this database and the extent of its source materials require a greatly expanded discussion of search options, as well as examples of the use of these options. 
1. New Search/Basic Search is the default search screen, with links to Search Options (which offers "Apply related words" and "... search full text of the articles" and various limits), Advanced Search (which is Basic Search plus Search Options), Visual Search (which presents results with subject headings for limiting), and Search History. Phrase searching is the default, along with the Boolean search options: AND; OR; and NOT. Fielded searching (e.g., author, molecular formula, name of substance, source collection, title words, etc.) is also available. Click "Advanced Search", which shows a "Field Codes" link just above the search box for a listing of Field Code tags.

Example Ia: A New Search (Basic Search) for 'Dioxin' retrieves over 17,000 results.

Example 1b: Entering Dioxin as a keyword and then clicking Advanced Search offers the additional options of including "Apply related words" and "Also search within the full text of the articles", which now retrieves over 21,000 results. Adding a Field Code (TI dioxin) now retrieves the 12,000 results that have "dioxin" as a title word. Results can be refined by faceted options in the left margin: Full Text; PDF Full Text; Subject; and Publication. There is also a "date" scale for additional limiting.

Example 1c: A search for "Dioxin" in Visual Search provides an interesting "Group Results" list of 250 results with a choice of either refining with subject terms or providing a list of publications.

2. EXPERTIndex defaults to a searchable/browsable listing of more than 2.25 million names \& synonyms. Clicking on a compound name/synonym generates a search of the CHIL compound ID number, within Search History, and provides options to View Results (a listing of documents), View Details, or Edit (which allows access to Search Options).

Example 2: A browse/search for "Dioxin" in EXPERTindex retrieves 4920 results. The default is both "related words" and "full text", and the results can by refined by faceted options in the left margin. The EXPERTINdex search searches the UI field for the UI code. These documents are "about" dioxin, as opposed to being documents containing the word "dioxin".

3. Collections is a browsable listing of all the collections in CHIL. Clicking on a collection name generates a search which allows adding additional search terms and limits results to that collection. 
Example 3: A Collections search for "CPDB - Carcinogenic Potency Database AND dioxin" "(where dioxin is searched as a text word) retrieves three results, which, in this case, provide extensive experimental detail on carcinogenicity.

4. ChemID Enhanced is a searchable/browsable listing of names and synonyms. Clicking on a compound name displays a structure diagram, basic physical properties, synonyms, classification codes, RTECS descriptors, and Chemical and Physical Properties from the Hazardous Substances Data Bank. Clicking on the name of a substance in the ChemID Enhanced record generates an EXPERTIndex search.

Example 4: A search for "Dioxin" in ChemID Enhanced retrieves a record with the chemical structure, molecular formula, CAS $R N$, Synonyms, etc., and HSDB chemical and physical properties. Clicking on the chemical name link generates an EXPERTindex search, that can be modified with additional criteria, such as "plastics", to limit retrieval to three results.

5. More. Clicking on Indexes offers the option to browse various indexes (e.g., Category, Collection, Document Type, Published Date) and add search terms.

7.4.2.2 Hazards Resources - Freely Available. TOXNET. The TOXNET $^{40}$ fact sheet states that it "is a group of databases covering chemicals and drugs, diseases and the environment, environmental health, occupational safety and health, poisoning, risk assessment and regulations, and toxicology" and that information in the TOXNET databases covers:

- specific chemicals, mixtures, and products;

- chemical nomenclature;

- unknown chemicals;

- special toxic effects of chemicals in humans and/or animals; and

- citations from the scientific literature.

The primary TOXNET databases of interest to chemists are ChemIDplus, HSDB, Toxline, and the Household Products Database.

ChemIDplus $^{41}$ is a web-based search system that serves as the chemical index for TOXNET. ChemIDplus records provide structure descriptors (InChI, Smiles), chemical synonyms, toxicity values, physical properties and Locator Code links to a wide variety of other government agency 
sponsored files (NLM, FDA, EPA, Superlist Locator, etc.) and databases (PubMed, PubChem, etc.).

At the time of this writing, the database contains over 388,000 chemical records, of which over 295,000 include chemical structure diagrams, and is searchable by Name, Synonym, CAS Registry Number, Molecular Formula, Classification Code, Locator Code, Structure, Toxicity, and/or Physical properties.

The ChemIDplus Lite version is available for simplified Name and CAS RN searching without the need for plugins or applets. ChemIDplus Advanced allows searching for chemical structures and substructures (MarvinSketch), toxicity, and physical properties. An enhanced structure display is also available by clicking the Enlarge Structure link.

The Hazardous Substances Data Bank (HSDB) ${ }^{42}$ is the most comprehensive online resource for hazardous substances. It is freely available and provides peer-reviewed toxicological information for over 5,400 potentially hazardous chemicals. HSDB records for individual chemicals provide data in the following categories.

- Human Health Effects.

- Emergency Medical Treatment.

- Animal Toxicity Studies.

- Metabolism/Pharmacokinetics.

- Pharmacology.

- Environmental Fate/Exposure.

- Environmental Standards \& Regulations.

- Chemical/Physical Properties.

- Chemical Safety \& Handling.

- Occupational Exposure Standards.

- Manufacturing/Use Information.

- Laboratory Methods.

- Special References.

- Synonyms and Identifiers.

- Administrative Information.

A unique feature of the HSDB are the extensive references to a core set of books, government documents, technical reports, and the primary journal literature.

HSDB search terms include chemical names and name fragments, synonyms, CAS RN. The text is keyword searchable (e.g., chromium compounds, kidney failure, oil spill, etc.). Searching for a specific 
chemical (e.g., Benzene) displays a link to the primary Benzene record as well as links to other records that mention Benzene. Clicking the primary Benzene link displays the "Human Health Effects" section, with the full table of contents in the left margin. If you click on one of the other records or perform a keyword searching for a topic, the default display will be the Best Section (i.e., section with the highest term frequency).

TOXLINE $^{43}$ is a bibliographic database covering the biochemical, pharmacological, physiological, and toxicological effects of drugs and other chemicals. It currently has over 4 million records with abstracts, indexing terms and CAS Registry Numbers. TOXLINE sources include PubMed, reports and archival material from other government agencies, and other TOXNET databases. Search results offer: relevancy ranking; sorting; and downloading options.

The Household Products Database ${ }^{44}$ provides information on potential health effects of chemicals found in more than 10,000 products used inside and around the home. Users can browse product categories (e.g., Pesticides), sub-categories (e.g., Insecticide), or types (e.g., ants), which then display an alphabetical listing of product names (e.g., Ant Stop Orthene Fire Ant Killer); or, one can scan alphabetical lists of product names (e.g., Alumaseal), types of products (e.g., Antifreeze), manufacturers (Airwick Industries), and ingredients (e.g., Acetone).

There are also both a quick search and an advanced search option for product name, type, manufacturer, ingredient (searchable by chemical name or CAS Registry Number), and health effects, all of which can also be limited by product category.

Clicking on the Product Name for a record displays product information, including a customer service phone number and links to "products with similar usage", and manufacturer information, including "Related Items: Products by this Manufacturer" and health effects, handling and disposal, and ingredients information from the manufacturer's Material Safety Data Sheet (MSDS), as well as a link to the complete MSDS. It is also possible to highlight terms and initiate a search in the TOXNET database.

The International Programme on Chemical Safety (IPCS), ${ }^{45}$ in partnership with CCOHS, was developed to establish a scientific rationale for management of chemicals, and to ensure chemical safety of both humans and the environment. The IPCS publication program (http:/) www.inchem.org/) covers both natural and manufactured chemicals and exposure situations ranging from chemicals in the natural environment to their extraction or synthesis, industrial production, transport, use, and disposal. 
The Nanomaterial-Biological Interactions Knowledgebase (NBIK) ${ }^{46}$ is designed to provide a scientific basis for understanding the effects of nanomaterial exposure in biological systems. The NBIK collects information on characteristics of nanomaterials (purity, size, shape, charge, composition, functionalization, agglomeration state); methods of synthesis; and beneficial, benign or deleterious nanomaterial-biological interactions at the organism, cellular, and molecular level. The NBIK database will allow for accurate definition of nanomaterial structureactivity relationships, and these representations can be used to predict nanomaterial properties in the absence of empirical data.

The KNOWLEDGEBASE link provides access to both a Nanomaterial Library and a Biological Interactions Database, which have dropdown menus for searching by material type, shape, core, charge, surface Chemistry, size $(\min / \max )$, and dendrimer generation. Additional information links are provided for the following.

- Goals and Objectives of the NBI.

- Biological Implications of Nanotechnology.

- Organizational Chart.

- Frequently Asked Questions.

\section{ACToR (Aggregated Computational Toxicology Resource) ${ }^{47}$ is a} publicly available database focused on high and medium production volume industrial chemicals, pesticides (both active and inert ingredients), and potential ground and drinking water contaminants. It provides access to over 1,000 resources and information on chemical toxicity and potential chemical risks for over 500,000 chemicals. ACToR is searchable by chemical names (keyword or exact), CAS Registry Numbers, and chemical structures searched as both substructures and similarity, via a ChemAxon structure editor.

Search results (after clicking the "details" link) include basic physical property data, synonyms, toxicology data (hazard, acute toxicity, chronic toxicity, carcinogenicity, genetic toxicity, ecotoxicity), category data (risk management, exposure data), and a list of external databases searched by name or CAS RN.

ChemSpider $^{48}$ is a freely available database, based on chemical structures, that provides information on over 26 million de-duplicated compounds derived from over 400 sources. These sources include a wide variety of government databases, chemical supplier catalogs, and academic and commercial websites. Each of the listed sources ${ }^{49}$ has a brief pop-up description, with the full record providing a web link to the 
source. ChemSpider augments the default information provided by these sources with a very wide variety of additional of data, including:

- Properties/Experimental: Presents flammability, toxicity, and safety data.

- RSC Databases: Click on "Laboratory Hazards Bulletin" for journal article titles, author names, and article DOIs.

- Medical Subject Headings Classification: MeSH heading and PubMed terms and heading to which the substance maps.

Bear in mind that: "Before you do anything with ChemSpider it is important to remember that it isn't yet either perfect or exhaustive."50

ChemSpider search options include:

1. Simple: Systematic name; synonym; trade name; Registry Number; SMILES, InChI, or CSID, with additional options for single/ multi-component, isotopically labeled, and additional filters.

2. Structure: Upload a structure or image file; convert name, SMILES, InChI, or ChemSpider ID to structure; or draw a structure (Accelrys JDraw, Elemental, ACD/Labs SDA, Ketcher, JME, or JChempaint). Searches can be exact, substructure, or similarity, with additional options for exact match, all tautomers, same skeleton (including or excluding $\mathrm{H}$ ), and all isomers.

3. Advanced: Search by structure, identifier, elements, properties, calculated properties, data source, etc., and by LASSO (Ligand Activity in Surface Similarity Order) similarity.

4. Search History: Each search is assigned an internal ID number, date/time started and updated, status, progress, predicate (search string), and system message.

\subsubsection{Conclusion}

This section provides an overview of significant resources for hazardous chemicals and chemical products. Given the growing importance of this problem in the United States, new publications and resources will surely continue to appear on a regular basis. Thieme Medical Publishers has prepared a comprehensive listing of hazard resources for "the different hazardous properties that Copy Editors should take into consideration when annotating experimental procedures", ${ }^{51}$ which also includes both US, UK and European legislation related to hazards and laboratory safety. ${ }^{51}$

Sources for continuing education are weekly reading of Chemical \& Engineering News and maintaining awareness of new publications and 
databases (e.g., Exposure Science in the 21st Century: A Vision and a Strategy, ${ }^{52}$ ChemHat.org, ${ }^{53}$ and NOAA's Chemical Reactivity Worksheet. $^{54}$

\section{REFERENCES}

1. Aldrich Chemistry, http:/www.sigmaaldrich.com/chemistry.html, Accessed April 16, 2013.

2. Chemical Book, http:/www.chemicalbook.com/ProductIndex_ EN.aspx, Accessed April 16, 2013.

3. ChemNet : Global Chemical Network, http:/www.chemnet.com/, Accessed April 16, 2013.

4. (a) MatWeb ... Source for Materials Information, http: www.matweb.com/, Accessed April 16, 2013; (b) Benefits of registering with MatWeb, http://www.matweb.com/membership/ benefits.aspx, Accessed April 16, 2013.

5. ChemExper Chemical Directory, http:/www.chemexper.com/, Accessed April 16, 2013.

6. Chemical Suppliers - CHEMCATS - Find commercially available chemicals, pricing, and supplier contact information, http:// www.cas.org/expertise/cascontent/chemcats.html, Accessed April $16,2013$.

7. Reaxys, https://www.reaxys.com/info/, Accessed April 16, 2013.

8. eMolecules, http://www.emolecules.com/, Accessed April 16, 2013.

9. American Chemical Society. The Chemical Professional's Code of Conduct, http://portal.acs.org/portal/PublicWebSite/careers/ profdev/ethics/CNBP_023290, Accessed May 24, 2013.

10. Chemical Safety: Nitric Oxide at High Pressure, Chemical \& Engineering News, 2012, 90, 6, http:/cen.acs.org/articles/90/i5/ Chemical-Safety-Nitric-Oxide-High.html, Accessed May 24, 2013.

11. Central Science: The Safety Zone, http://cenblog.org/the-safetyzone/, Accessed April 17, 2013.

12. Creating Safety Cultures in Academic Institutions, www.acs.org/ safety, Accessed April 16, 2013.

13. Material safety data sheet. Wikipedia, the Free Encyclopedia, http:/ en.wikipedia.org/wiki/Material_safety_data_sheet, Accessed April 17, 2013.

14. Links to materials safety data sheets are available in commercial chemical supplier catalogs, some of which are described in Section 7.2.

15. National Research Council of the National Academies, Prudent practices in the laboratory: Handling and management of chemical 
hazards, National Academies Press, Washington, D. C., 2011. http:/ www.nap.edu/catalog.php?record_id=12654, Accessed May 24, 2013.

16. Chemical safety and toxicology searches. In Chemical Information Sources, http:/en.wikibooks.org/wiki/Chemical_Information_ Sources/Chemical_Safety_Searches, Accessed April 17, 2013.

17. CAS Registry and CAS Registry Number FAQs. CAS (Chemical Abstracts Service), http:/www.cas.org/content/chemical-substances faqs, Accessed May 25, 2013.

18. P. Hunt. Letters: Assessing Chemical Risk: Societies Offer Expertise, Science, 2011, 131, 1136, http://www.sciencemag.org/content/ 331/6021/1136.1.full.pdf, Accessed May 24, 2013.

19. Toxic Substances Control Act of 1976, Wikipedia, the Free Encyclopedia, http:/en.wikipedia.org/wiki/Toxic_Substances_Control_ Act_of_1976, Accessed May 22, 2013.

20. TSCA Chemical Substance Inventory, http:/www.epa.gov/oppt/ existingchemicals/pubs/tscainventory/index.html, Accessed May 22, 2013.

21. P. Callahan, S. Roe. Big Tobacco wins fire marshals as allies in flame retardant push, Chicago Tribune Watchdog, May 8, 2012, http:/ www.chicagotribune.com/news/watchdog/flames/ct-metflames-tobacco-20120508,0,3332088.story, Accessed May 24, 2013.

22. C. Hogue. New Tools for Risk Assessment, Chemical \& Engineering News, 2012, 90, 32, http://cen.acs.org/articles/90/i24/New-ToolsRisk-Assessment.html, Accessed May 24, 2013.

23. T. Masciangioli, J. Alper. Challenges in characterizing small particles: exploring particles from the nano- to microscales: A workshop summary, National Academies Press, 2012, http://www.nap.edu/ catalog.php?record_id=13317, Accessed May 24, 2013.

24. R. F. Service. Can high-speed tests sort out which nanomaterials are safe? Science, 2008, 321,1036, doi: 10.1126/science.321.5892.1036, Accessed May 24, 2013.

25. S. George, T. Xia, R. Rallo, Y. Zhao, Z. Ji, S. Lin, X. Wang, H. Zhang, B. France, D. Schoenfeld, R. Damoiseaux, R. Liu, S. Lin, K. A. Bradley, Y. Cohen and A. E. Nel, ACS Nano, 2011, 5, 1805, doi: 10.1021/nn102734s, Accessed May 24, 2013.

26. National Research Council. A research strategy for environmental, health, and safety aspects of engineered nanomaterials. National Academies Press, 2012, http:/www.nap.edu/catalog.php?record_ $\mathrm{id}=13347$, Accessed May 24, 2013.

27. ToxTown - Nanoparticles, http:/www.toxtown.nlm.nih.gov/text version/chemicals.php?id=67, Accessed May 22, 2013. 
28. Endocrine disruptor, Wikipedia, the Free Encyclopedia, http://en. wikipedia.org/wiki/Endocrine_disruptor, Accessed May 22, 2013.

29. C. Z. Yang, S. I. Ganiger, V. C. Jordan, D. J. Klein, G. D. Bittner. Environ Health Perspect, 2011, 119, 989, http:/ehp03.niehs.nih.gov article info:doi\%2F10.1289\%2Fehp.1003220, Accessed May 24, 2013.

30. C. A. Frye, E. Bo, G. Calamandrei, L. Calzà, F. Dessi-Fulgheri, M. Fernández, L. Fusani, O. Kah, M. Kajta, Y. Le Page, H. B. Patisaul, A. Venerosi, A. K. Wojtowicz and G. C. Panzica. J. Neuroendocrinol, 2012, 24, 144, doi: 10.1111/j.1365-2826.2011. 02229.x., Accessed May 24, 2013.

31. PubMed, http://www.ncbi.nlm.nih.gov/sites/entrez?otool=caitlib, Accessed May 22, 2013.

32. Federal Register - The Daily Journal of the U.S. Government, https:/www.federalregister.gov/, Accessed May 22, 2013.

33. Regulated Chemicals - CHEMLIST, http://www.cas.org/content regulated-chemicals, Accessed May 22, 2013.

34. P. Urben. Bretherick's Handbook of Reactive Chemical Hazards, 7 th ed. Amsterdam; London: Elsevier, 2006. Work consists of two volumes: v.1: Introduction; Reactive chemical hazards; Specific Chemicals (in formula order), and v.2: Classes, Groups and Topics (in alphabetical order).

35. N. Langeman, R. J. Alaimo, F. Fung. Hazmat Navigator, http:/ www.elsevierdirect.com/hazmatnavigator/home.html, Accessed May 25, 2013. Hazmat Navigator is a chemical safety database based on Bretherick's Handbook of Reactive Chemical Hazards (subscription required/free trial available).

36. R. J. Lewis. Sax's Dangerous Properties of Industrial Materials, 12th ed., New York: John Wiley \& Sons, 2013. http:/onlinelibrary. wiley.com/book/10.1002/0471701343, Accessed May 25, 2013.

37. Patty's Industrial Hygiene and Toxicology Online, http:/ onlinelibrary.wiley.com/book/10.1002/0471125474, Accessed May 25, 2013. Includes Patty's Industrial Hygiene, $6^{\text {th }}$ Ed., edited by Vernon E. Rose (2011) and Patty's Toxicology, $6^{\text {th }}$ ed., edited by Eula Bingham (2012).

38. S. Gangolli. Dictionary of Substances and their Effects (DOSE), 2nd ed. Cambridge: Royal Society of Chemistry, 1999. Available online thru Dialog (309), Knovel, and the RSC.; About DOSE, http:/ www.rsc.org/Publishing/CurrentAwareness/DOSE/About.asp, Accessed May 25, 2013.

39. CHIL: Chemical Hazard Information Library (subscription required/free trial available), EBSCO Discovery Service, http:/ 
www.ebscohost.com/ biomedical-libraries/chemical-hazard-informationlibrary, Accessed May 25, 2013.

40. (a) TOXNET: TOXicology Data NETwork, National Library of Medicine Fact Sheet: http:/www.nlm.nih.gov/pubs/factsheets/ toxnetfs.html Database links: http:/toxnet.nlm.nih.gov/, Accessed May 25, 2013. (b) TOXNET and Beyond: Using the National Library of Medicine's Environmental Health and Toxicology Portal, National Library of Medicine, 2011, http:/sis.nlm.nih.gov/ enviro/toxnetmanualfeb2011.pdf, Accessed May 25, 2013.

41. ChemIDplus, National Library of Medicine Factsheet: http:/www.nlm.nih.gov/pubs/factsheets/chemidplusfs.html, Accessed May 25, 2013. Database (Lite): http:/chem.sis.nlm.nih.gov/ chemidplus/chemidlite.jsp, Accessed May 25, 2013. Database (Advanced): http://chem.sis.nlm.nih.gov/chemidplus/, Accessed May 25, 2013.

42. Hazardous Substances Data Bank (HSDB), National Library of Medicine Fact Sheet: http:/www.nlm.nih.gov/pubs/factsheets/ hsdbfs.html, Accessed May 25, 2013. Data Bank: http:/ toxnet.nlm.nih.gov/cgi-bin/sis/htmlgen?HSDB, Accessed May 25, 2013.

43. TOXLINE, National Library of Medicine Factsheet: http:/ www.nlm.nih.gov/pubs/factsheets/toxlinfs.html, Accessed May 25, 2013. Database: http://toxnet.nlm.nih.gov/cgi-bin/sis/ htmlgen?TOXLINE, Accessed May 25, 2013.

44. Household Products Database, National Library of MedicineFactsheet: http:/www.nlm.nih.gov/pubs/factsheets/ householdproducts.html, Accessed May 25, 2013. Database: http:/ householdproducts.nlm.nih.gov/, Accessed May 25, 2013.

45. International Programme on Chemical Safety (IPCS), World Health Organization, http:/www.who.int/ipcs/en/, Accessed May 25, 2013.

46. Harper, Stacy. Nanomaterial-Biological Interactions Knowledgebase (NBIK), http://nbi.oregonstate.edu/, Accessed May 25, 2013.

47. ACToR - Aggregated Computational Toxicology Resource, Environmental Protection Agency, http:/actor.epa.gov/actor/faces/ ACToRHome.jsp, Accessed May 25, 2013.

48. ChemSpider; the free chemical database, Royal Society of Chemistry, http:/www.chemspider.com , Accessed May 25, 2013.

49. ChemSpider; the free chemical database - Data Sources, Royal Society of Chemistry, http:/www.chemspider.com/DataSources. aspx, Accessed May 25, 2013. 
50. ChemSpider; the free chemical database - Getting Started, Royal Society of Chemistry, http:/www.chemspider.com/GettingStarted. aspx, Accessed May 25, 2013.

51. Hazard Information for Science of Synthesis, Thieme Medical Publishers, http:/www.thieme-chemistry.com/fileadmin/Thieme SoS/pdf/hazard2.pdf, Accessed May 25, 2013.

52. Exposure Science in the 21st Century: A Vision and a Strategy. National Research Council, 2012, http:/www.nap.edu/openbook. php?record_id=13507\&page=1, Accessed May 25, 2013.

53. ChemHat.org - Chemical Hazard and Alternatives Toolbox, Communications Workers of America and BlueGreen Alliance, http://www.chemhat.org/, Accessed May 25, 2013.

54. National Oceanic and Atmospheric Administration (NOAA) Office of Response and Restoration, Chemical Reactivity Worksheet, http://response.restoration.noaa.gov/chemaids/react.html, Accessed May 25, 2013. 